・研究报告・

\title{
内蒙古昆虫物种多样性分布格局及其机制
}

\author{
张宇冯 刚* \\ (蒙古高原生态学与资源利用教育部重点实验室, 省部共建草地生态学国家重点实验室培育基地, \\ 内蒙古大学生态与环境学院, 呼和浩特 010021)
}

\begin{abstract}
摘要: 物种多样性的地理分布格局及其机制是宏生态学和生物地理学的核心问题之一。区域尺度与局域尺度的影 响因素, 如温度、降水、海拔变化、生境过滤、捕食、竞争与互惠等, 共同影响昆虫物种多样性的分布格局。然 而, 迄今为止少有研究同时讨论不同尺度驱动因子对昆虫多样性地理分布格局的影响。本文基于内蒙古自治区86 个旗县的昆虫多样性数据, 结合各地年平均气温、年降水量、古气候变化、海拔变化及植物多样性, 探讨昆虫物 种多样性分布格局及其主要驱动因子。结果发现内蒙古昆虫多样性主要受到植物多样性与海拔变化的影响, 而气 候因子对昆虫物种多样性的影响并不大。这一结果表明种间关系(食物多样性)与生境异质性可能对内蒙古昆虫多 样性的分布格局起着主导作用。
\end{abstract}

关键词：昆虫物种多样性; 生境异质性; 种间关系; 气候因子; 海拔变化; 植物多样性

\section{Distribution pattern and mechanism of insect species diversity in Inner Mongolia}

\author{
Yu Zhang, Gang Feng* \\ Ministry of Education Key Laboratory of Ecology and Resource Use of the Mongolian Plateau \& Inner Mongolia Key \\ Laboratory of Grassland Ecology, School of Ecology and Environment, Inner Mongolia University, Hohhot 010021
}

\begin{abstract}
How species are distributed geographically and what drives these distributions remain core issues in macroecology and biogeography. Both regional and local scale factors such as temperature, precipitation, altitudinal range, habitat filtering, predation, competition and reciprocity affect the large-scale distribution pattern of insect species diversity. However, few studies have simultaneously discussed the effects of these multi-scale drivers on the geographical distribution of insect diversity. Using insect diversity data from 86 counties in Inner Mongolia Autonomous Region, we assessed the distribution patterns and main drivers of insect species diversity. We included mean annual temperature, mean annual precipitation, paleoclimate change, altitudinal range and plant diversity as predictors. Plant diversity and altitudinal range influenced insect diversity in Inner Mongolia while climatic factors had less influence. Our results show that interspecific relationships (food diversity) and habitat heterogeneity may play a crucial role in shaping distribution patterns of insect diversity in Inner Mongolia.
\end{abstract}

Key words: insect species diversity; habitat heterogeneity; interspecific interactions; climatic factor; altitudinal range; plant diversity

物种多样性的地理分布格局及其机制是生态 学及生物地理学的核心问题之一(Currie, 1991; Brown, 2014; Fine, 2015)。其中，区域尺度的物种形 成、灭绝及迁徙，与局域水平的生境过滤、种间关 系及扩散限制共同影响物种多样性的地理分布格
局(Fine, 2015; Qu et al, 2015; Svenning et al, 2015)。 已有研究发现中国亚热带森林中树木多样性显著 促进昆虫多样性(Schuldt et al, 2010)。中国蚂蚁丰富 度与最冷月温度、年降水量和年温度变化显著相关 (沈梦伟等, 2016)。然而, 较少有研究同时比较不同 
尺度影响因素对大尺度昆虫多样性分布格局的影响。 近年来, 许多学者提出了关于物种多样性分布 格局的假说, 包括水热动态假说(water-energy dynamic hypothesis)、寒冷忍耐假说(freezing tolerance hypothesis)、种库假说(species pool hypothesis)与植 物生长势假说(plant vigor hypothesis)。O'Brien最早 提出的水热动态假说, 其主要观点是物种多样性的 大尺度格局是由能量和水分共同控制的(O’Brien, 1998; O’Brien et al, 2000)。寒冷忍耐假说的主要观 点是在寒冷地区, 由于很多物种不能忍受冬季的低 温而无法生存, 随着冬季温度的降低, 物种多样性 逐渐减少(Hawkins, 2001)。种库假说认为, 一个群 落的物种多样性不仅与环境条件和生态过程(如竞 争、捕食)有关, 也受区域种库的限制。区域种库是 指一个地区可进入某一群落的潜在物种数量, 它由 地史过程(如冰期、地质年代)和区域过程(物种形 成、迁移扩散以及消亡)所决定(Zobel, 1997)。而植 物生长势假说的观点是昆虫更倾向于取食并生活 在植物生长旺盛且茂密的地方 (Price，1991)。 Harrison和Cornell (2008)认为, 群落的物种多样性 往往受到局域气候、干扰以及区域多样性的控制, 而区域生物多样性主要受区域历史过程与环境(包 括现代环境和古环境)控制。

昆虫是地球上较早出现的动物类群之一, 距今 约有4亿8千万年(Misof et al, 2014)。作为地球上多 样性最为丰富的多细胞生物类群, 迄今已经发现 100多万种, 并且仍有更多未描述的物种(Diniz-Filho et al, 2010)。与此同时, 昆虫的生物多样性保护对于 陆地生态系统多样性维持的生态学及进化学过程 有重要作用(Thomas et al, 2008; Diniz-Filho et al, 2010)。然 ，由于分类信息及地理分布信息的缺乏, 昆虫生物地理学分布格局的相关研究一直以来较 为贵乏(Diniz-Filho et al, 2010)。

内蒙古 $\left(37^{\circ} 24^{\prime}-53^{\circ} 23^{\prime} \mathrm{N}, 97^{\circ} 12^{\prime}-126^{\circ} 04^{\prime} \mathrm{E}\right)$ 地 域辽阔, 经度范围较大, 海拔在532-1,725 m之间, 包括平原、高原和山地 3 种典型地貌(能乃扎布, 1999)。年平均降水量 40-450 mm, 年平均气温-2至 $6^{\circ} \mathrm{C}$, 因此内蒙古由东向西形成了森林、草甸草原、 典型草原、荒漠草原和荒漠等植被类型。复杂多样 的地理条件与丰富的植被类型维持着较高的生物 多样性, 如内蒙古有维管植物 2,447种, 鸟类467种, 哺乳动物149种, 昆虫6,346种, 这些都为生物多样
性大尺度的研究提供了一个良好的平台(能乃扎布, 1999; 旭日干, 2007, 2015, 2016a, b; 赵一之和赵利 清, 2014)。

内蒙古已有的昆虫多样性研究大多集中在区 域的物种多样性描述与区系分析，尚未有研究从整 个内蒙古自治区尺度讨论昆虫多样性的分布格局 及其影响因素。本研究首次将内蒙古昆虫物种多样 性与现代气候因子、古气候变化、海拔变化及植物 多样性等影响因子联系起来，从而分析比较不同尺 度的因子对昆虫多样性分布格局的相对作用。

\section{材料与方法}

\section{1 昆虫及植物分布数据}

昆虫分布数据来自《内蒙古昆虫》，该书包括 了内蒙古自治区分布的昆虫纲和蛛形纲动物 28 目 386科6,346种，其中昆虫25目341科5,982种(能乃扎 布，1999)。我们搜集了有旗县分布信息的物种，最 终包括昆虫类及蜘蛛类动物 25 目 341 科 2,505 种, 分 布在内蒙古的 86 个旗县区。植物分布数据来自中国 维管植物分布数据库，该数据库中植物分布信息来 自《中国植物志》、各省及区域植物志、植物标本 数据等。

\section{2 影响因子}

本研究选择的昆虫物种多样性影响因子有现 代气候(年均温及年降水)、古气候变化(末次最大冰 期到现在的温度变化及降水变化)、海拔变化与植物 多样性。从WorldClim收集了海拔变化信息及气候 变量, 即年平均温度 (mean annual temperature, MAT)、年平均降水量(mean annual precipitation, $\mathrm{MAP})$ 、末次最大冰期温度 $\left(\mathrm{MAT}_{\mathrm{LGM}}\right)$ 、末次最大冰 期降水量 $\left(\mathrm{MAP}_{\mathrm{LGM}}\right)$ (Hijmans et al, 2005)。以海拔变 化梯度作为环境异质性的代表(Stein et al, 2014)。 MAT $_{\mathrm{LGM}}$ 和MAP $\mathrm{LGM}_{\mathrm{LG}}$ 来自于CCSM3 (Otto-Bliesner et al, 2006)和MIROC3.2 (Hasumi \& Emori, 2004)两个 模型的平均值, 然后用现代气候变量值减去末次最 大冰期气候变量值来表示温度及降水的变化 (Sandel et al, 2011)。气候数据及海拔数据的精度为 $0.0417^{\circ}$ ，其中 MAT 和 MAP 是 1960-1990年的平均 值。计算了每个旗县的平均值来代表该旗县的气候 变量值, 同时计算了每个旗县的海拔变化范围。

\section{3 数据分析}

首先对昆虫和植物的物种多样性进行对数转 
换以获得正态分布残差。所有的因变量和独立变量 都进行了标准化 (平均值 $=0$, 标准差 $=1$ ), 以使回 归系数具有可比性。使用最小二乘法(ordinary least squares)模型来评估昆虫多样性与每个影响因子之 间的关系。与此同时, 为了控制变量之间空间自相 关的影响, 采用空间自回归(simultaneous autoregressive)模型进行单变量分析。

为了得到对昆虫物种多样性分布格局解释率 最高的变量组合，利用随机森林模型对昆虫物种多 样性与气候因子、植物多样性、海拔变化梯度的相 关性做了分析。因为随机森林模型可以有效地处理 变量之间的共线性问题, 并且不要求数据遵循严格 的假设，例如同方差性和误差正态性 (Breiman, 2001)。对于每个组合变量, 随机森林模型将运行 1,000 次, 每次将数据随机分开, 即 $50 \%$ 的训练数据 和 $50 \%$ 的评估数据，计算预测值和观测值之间的 Pearson相关性。为了检查哪些变量总是在最佳组合 中出现，选择了 4 个Pearson相关性最高的组合。

\section{结果}

\section{1 单因素线性分析}

单因素最小二乘法模型的结果显示昆虫物种
多样性与植物多样性、海拔变化梯度均呈极显著正 相关关系 $\left(r^{2}\right.$ 的值分别为 0.16 和 0.11$)$; 昆虫物种多样 性与古降水变化(末次最大冰期到现在的降水变化) 和年平均气温均呈显著负相关关系 $\left(r^{2}\right.$ 的值均为 0.04); 昆虫物种多样性与古温度变化(末次最大冰 期到现在的温度变化)和年降水量没有显著相关关 系(图1和表1)。空间自回归模型的结果与单因素最 小二乘法模型的结果相似, 即与昆虫物种多样性相 关性最高的是植物多样性与海拔变化(表1)。

\section{2 多因素随机森林分析结果}

随机森林模型的结果显示与昆虫物种多样性 相关最高的 4 个变量组合中均包括植物多样性与古 降水变化。与此同时, 海拔变化出现在其中的 3 个组 合(图2)。该结果表明内蒙古昆虫多样性分布格局同 时受到植物多样性、古降水变化及海拔变化的影响。

\section{讨论}

生物多样性的地理分布格局受不同时空尺度 因素的共同影响，包括局域的种间关系、生境过滤、 扩散限制，区域的物种形成、灭绝和迁徙，以及历 史气候变化(Fine, 2015; Svenning et al, 2015)。我们 的研究发现, 植物多样性、古降水变化及海拔变化

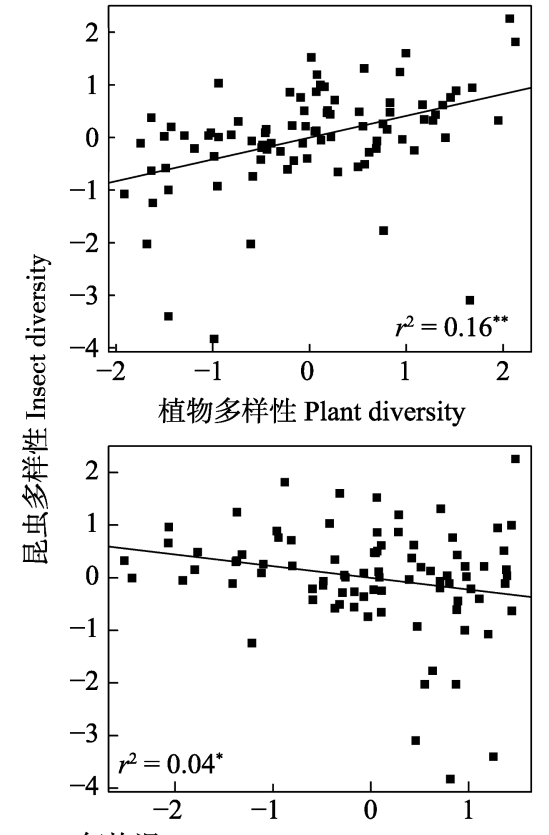

年均温 Mean annual temperature (MAT)

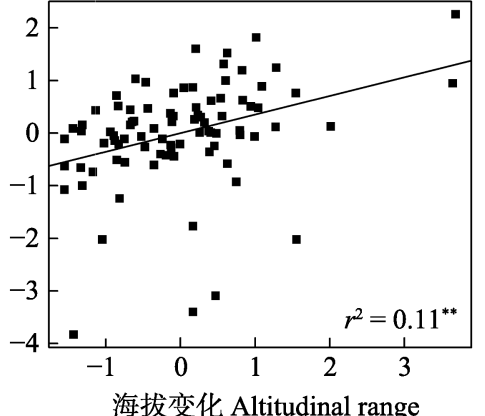

海拔变化 Altitudinal range

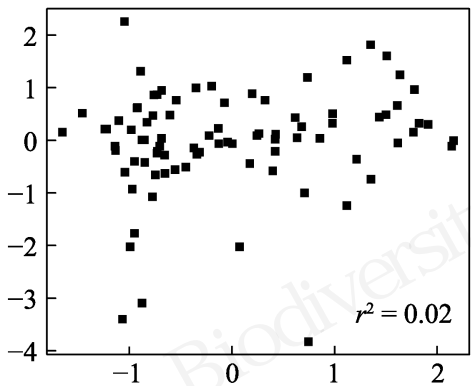

古温度变化 Anom MAT
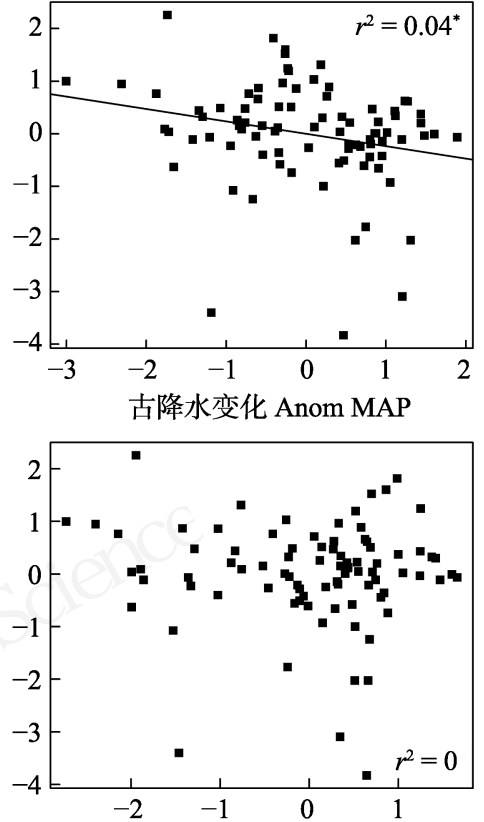

年降水量 Mean annual precipitation (MAP)

图1 昆虫多样性及其相关变量散点图。古降水变化与古温度变化是末次最大冰期到现代的降水和温度的变化。 $* \boldsymbol{P}<0.05, * *$ $P<0.01$ 。

Fig. 1 Scatter plots of insect species diversity and the related variables. Anom MAP and Anom MAT are the change of MAP/MAT between LGM and contemporary time. 
表1 最小二乘法(OLS)和空间自回归(SAR)模型单变量分 析结果

Table 1 Results of single-variable analysis by ordinary least squares (OLS) and simultaneous autoregressive (SAR) models

\begin{tabular}{lcccc}
\hline & coef $_{\mathrm{OLS}}$ & $r^{2}{ }_{\mathrm{OLS}}$ & coef $_{\mathrm{SAR}}$ & $r_{\text {SAR }}^{2}$ \\
\hline $\begin{array}{l}\text { 年平均气温 } \\
\text { Mean annual temperature (MAT) }\end{array}$ & -0.22 & $0.04^{*}$ & -0.24 & $0.08^{* *}$ \\
$\begin{array}{l}\text { 年降水量 } \\
\text { Mean annual precipitation (MAP) }\end{array}$ & -0.06 & 0 & -0.05 & 0 \\
$\begin{array}{l}\text { 古温度变化 } \\
\text { Anom MAT }\end{array}$ & 0.17 & 0.02 & 0.2 & $0.06^{* *}$ \\
$\begin{array}{l}\text { 古降水变化 } \\
\text { Anom MAP }\end{array}$ & -0.24 & $0.04^{*}$ & -0.23 & $0.07^{*}$ \\
$\begin{array}{l}\text { 植物多样性 } \\
\text { Plant diversity }\end{array}$ & 0.35 & $0.16^{* *}$ & 0.36 & $0.13^{* *}$ \\
海拔变化 & & & & \\
Altitudinal range & 0.42 & $0.11^{* *}$ & 0.41 & $0.18^{* *}$ \\
\hline
\end{tabular}

Anom MAT and Anom MAP are the change of MAT/MAP between LGM and contemporary time. ${ }^{*} P<0.05$, $* * P<0.01$.

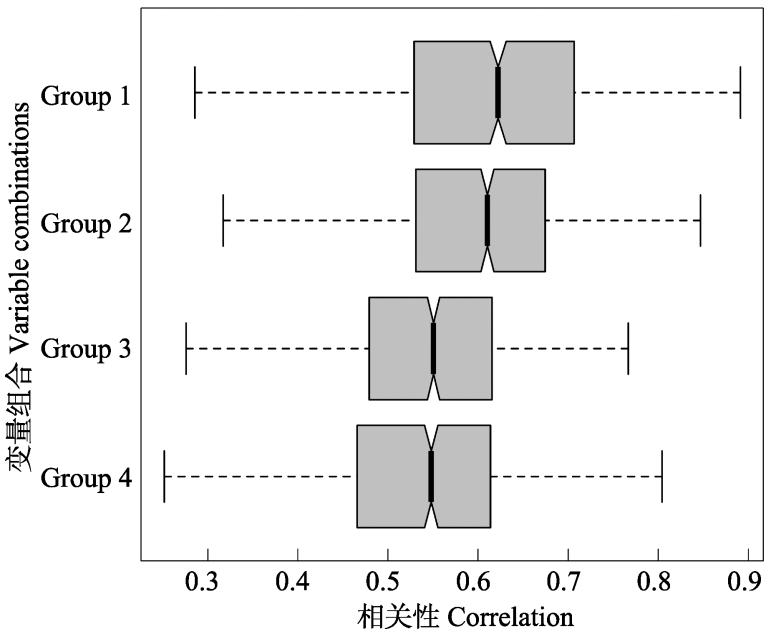

图2 随机森林模型得到的与昆虫物种多样性相关性最高的 4个组合。Group 1 为Anom MAP + Plant diversity, Group 2 为Anom MAP + Altitudinal range + Plant diversity, Group 3 为MAT + Anom MAP + Altitudinal range + Plant diversity, Group 4为MAP + Anom MAP + Altitudinal range + Plant diversity。Anom MAP表示古降水变化。

Fig. 2 Four combinations of variables most associated with insect species diversity obtained from the random forest model. Group 1 are Anom MAP and Plant diversity, Group 2 are Anom MAP, Altitudinal range and Plant diversity, Group 3 are MAT, Anom MAP, Altitudinal range and Plant diversity, Group 4 are MAP, Anom MAP, Altitudinal range and Plant diversity. Anom MAP is the change of MAP between LGM and contemporary time.

共同影响内蒙古昆虫物种多样性分布格局, 其中植 物多样性与昆虫多样性相关性最高, 强调了食物多 样性或生境多样性对大尺度昆虫物种多样性分布 格局的重要作用。

\section{1 植物多样性与昆虫物种多样性格局}

生物之间的相互作用是影响生物多样性地理 分布格局的重要因子之一(Wisz et al, 2013; Araújo \& Rozenfeld, 2014)。植物多样性与昆虫多样性的关 系是生态学研究中的基本问题之一(朱慧等, 2008; Zhang et al, 2016)。较高的植物多样性可以为昆虫提 供更多、更优质的食物，从而直接影响昆虫多样性; 与此同时，较高的植物多样性可以提供更多的生态 位空间、影响局域生境条件(土壤及空气温湿度), 从 而间接地影响昆虫多样性(朱慧等, 2008; Zhang et al, 2016)。

我们的研究结果发现内蒙古昆虫多样性与植 物多样性的相关性要高于与气候因素、海拔变化的 相关性，表明植物多样性对昆虫多样性分布格局有 重要的影响。这与前人的多数研究结果一致, 比如, 美国爱达荷州草原的植物多样性与昆虫多样性呈 正相关关系(Wenninger \& Inouye, 2008)。在中国热 带地区的云南西双版纳布龙自然保护区与海南鹦 哥岭自然保护区，根据植物多样性可以准确地预测 昆虫多样性(Zhang et al, 2016)。在巴拿马热带雨林 地区，植物多样性同样可以较好地估计昆虫多样性 的分布(Basset et al, 2012)。已有的研究还发现内蒙 古植物多样性能够较好地解释其鸟类多样性的分 布格局(Liang et al, 2018)。结合已有研究, 我们的结 果进一步证明生物之间相互作用或生境多样性可 能对昆虫多样性的地理分布格局有重要作用。

\section{2 海拔变化梯度与昆虫物种多样性格局}

生境异质性假说同样是生态学研究中的一个 基本问题。较高的生境异质性可以为生物提供更多 的生态位、避难所及物种形成的机会，从而进一步 促进较高的物种多样性的产生(Stein et al, 2014)。

Aranda和Graciolli (2015)对巴西热带稀树草原的研 究发现, 生境异质性即植被结构影响膜翅目昆虫群 落组成。Marini等(2010)对意大利阿尔卑斯山区域的 研究发现，木本植被的比例影响直翅目及蝴蝶的多 样性。

作为生境异质性的一个重要又易于测量的指 标, 海拔变化被广泛用于研究生境异质性与多样性 分布格局的关系(Tews et al, 2004)。南非蝗虫的多样 性分布显著地受到地形异质性的影响(Gebeyehu \& Samways, 2006)。坡度变化与意大利阿尔卑斯山区 域的昆虫与植物多样性显著正相关(Marini et al, 
2009)。海拔变化同样显著影响内蒙古鸟类多样性的 分布格局(Liang et al, 2018)。与前人的研究相似, 我 们的结果表明海拔变化能够较好地预测内蒙古昆 虫多样性分布格局, 从而验证了生境异质性假说对 内蒙古昆虫多样性地理分布格局的影响。

\section{3 气候因子与昆虫物种多样性格局}

气候因子, 包括当代气候及历史气候变化, 是 影响大尺度生物多样性分布格局的重要因素(Fine, 2015; Svenning et al, 2015)。我们的研究发现古降水 变化显著影响内蒙古昆虫多样性分布格局, 尤其是 随机森林分析结果显示古降水变化同植物多样性 一样, 出现在 4 个相关性最高的模型组合。并且古降 水变化越小的区域具有越高的昆虫多样性。这个发 现也与前人的研究相似, 比如, 澳大利亚热带地区 的历史冰期避难所拥有较高的昆虫物种多样性与 系统发育多样性(Boyer et al, 2016)。我们的结果进 一步证明了冰期避难所对生物多样性的保护作用。

\section{4 不足与展望}

本研究虽然提供了较为清楚的证据证明内蒙 古昆虫多样性受到植物多样性、古降水变化及海拔 变化的影响, 但仍有一些不足。如基于 《内蒙古昆 虫》的昆虫分布数据的准确性受到调查强度的影响, 基于植物志的植物多样性分布数据同样会受到调 查强度的影响, 旗县面积本身会对昆虫多样性有影 响。因此, 未来对昆虫多样性大尺度分布格局及机 制的研究可以基于统一的样方调查来进行, 从而控 制调查强度、取样面积等因素的影响。

\section{参考文献}

Aranda R, Graciolli G (2015) Spatial temporal distribution of the Hymenoptera in the Brazilian Savanna and the effects of habitat heterogeneity on these patterns. Journal of Insect Conservation, 19, 1173-1187.

Araújo MB, Rozenfeld A (2014) The geographic scaling of biotic interactions. Ecography, 37, 406-415.

Basset Y, Cizek L, Cuénoud P, Didham RK, Guilhaumon F, Missa O, Novotny V, Ødegaard F, Roslin T, Schmidl J, Tishechkin AK, Winchester NN, Roubik DW, Aberlenc HP, Bail J, Barrios H, Bridle JR, Castaño-Meneses G, Corbara B, Curletti G, da Rocha WD, Bakker DD, Delabie JH, Dejean A, Fagan LL, Floren A, Kitching RL, Medianero E, Miller SE, de Orivel EJ, Pollet M, Rapp M, Ribeiro SP, Roisin Y, Schmidt JB, Sørensen L, Leponce M (2012) Arthropod diversity in a tropical forest. Science, 338, 1481-1484.

Boyer SL, Markle TM, Baker CM, Luxbacher AM, Kozak KH
(2016) Historical refugia have shaped biogeographical patterns of species richness and phylogenetic diversity in mite harvestmen (Arachnida, Opiliones, Cyphophthalmi) endemic to the Australian Wet Tropics. Journal of Biogeography, 43, 1400-1411.

Breiman L (2001) Random forests. Machine Learning, 45, 5-32.

Brown JH (2014) Why are there so many species in the tropics? Journal of Biogeography, 41, 8-22.

Currie DJ (1991) Energy and large scale patterns of animal and plant species richness. The American Naturalist, 137, 27-49.

Diniz-Filho JAF, Marco PD, Hawkins BA (2010) Defying the curse of ignorance: Perspectives in insect macroecology and conservation biogeography. Insect Conservation and Diversity, 3, 172-179.

Fine P (2015) Ecological and evolutionary drivers of geographic variation in species diversity. Annual Review of Ecology, Evolution, and Systematics, 46, 369-392.

Gebeyehu S, Samways MJ (2006) Topographic heterogeneity plays a crucial role for grasshopper diversity in a Southern African megabiodiversity hotspot. Biodiversity and Conservation, 15, 231-244.

Harrison S, Cornell H (2008) Toward a better understanding of the regional causes of local community richness. Ecology Letters, 11, 969-979.

Hasumi H, Emori S (2004) K-1 Coupled Model (MIROC) description. K-1 Technical Report No.1. Center for Climate System Research, University of Tokyo, Tokyo.

Hawkins BA (2001) Ecology's oldest pattern? Trends in Ecology and Evolution, 16, 470.

Hijmans RJ, Cameron SE, Parra JL, Jones PG, Jarvis A (2005) Very high resolution interpolated climate surfaces for global land areas. International Journal of Climatology, 25, 1965-1978.

Liang CX, Feng G, Si XF, Mao LF, Yang GS, Svenning J-C, Yang J (2018) Bird species richness is associated with phylogenetic relatedness, plant species richness, and altitudinal range in Inner Mongolia. Ecology and Evolution, 8, 53-58.

Marini L, Fontana P, Battisti A, Gaston KJ (2010) Agricultural management, vegetation traits and landscape drive orthopteran and butterfly diversity in a grassland-forest mosaic: A multi-scale approach. Insect Conservation and Diversity, 2, 213-220.

Marini L, Paolo F, Sebastian K, Andrea B, Kevin G (2009) Impact of farm size and topography on plant and insect diversity of managed grasslands in the Alps. Biological Conservation, 142, 394-403.

Misof B, Liu SL, Meusemann K, Peters RS, Donath A, Mayer C, Frandsen PB, Ware J, Flouri T, Beutel RJ, Niehuis O, Petersen M, Izquierdo-Carrasco F, Wappler T, Rust J, Aberer AJ, Aspöck U, Aspöck H, Bartel D, Blanke A, Berger S, Böhm A, Buckley TR, Calcott B, Chen JQ, Friedrich F, Fukui M, Fujita M, Greve C, Grobe P, Gu SC, Huang Y, Jermiin LS, Kawahara AY, Krogmann L, Kubiak M, Lanfear R, Letsch H, Li YY, Li ZY, Li JG, Lu HR, Machida R, 
Mashimo Y, Kapli P, McKenna DD, Meng GL, Nakagaki Y, Navarrete-Heredia JL, Ott M, Ou YX, Pass G, Podsiadlowski L, Poh H, von Reumont BM, Schütte K, Sekiya K, Shimizu S, Slipinski A, Stamatakis A, Song WH, Su X, Szucsich NU, Tan MH, Tan XM, Tang M, Tang JB, Timelthaler G, Tomizuka S, Trautwein M, Tong XL, Uchifune T, Walzl MG, Wiegmann BM, Wilbrandt J, Wipfler B, Wong TKF, Wu Q, Wu GX, Xie YL, Yang SZ, Yang Q, Yeates DK, Yoshizawa K, Zhang Q, Zhang R, Zhang WW, Zhang YH, Zhao J, Zhou CR, Zhou LL, Ziesmann T, Zou SJ, Li YR, Xu X, Zhang Y, Yang HM, Wang J, Wang J, Kjer KM, Zhou X (2014) Phylogenomics resolves the timing and pattern of insect evolution. Science, 346, 763-767.

Nonnaizab (1999) Insects of Inner Mongolia, China. Inner Mongolia People's Publishing House, Hohhot. (in Chinese) [能乃扎布 (1999) 内蒙古昆虫. 内蒙古人民出版社, 呼 和浩特.]

O’Brien EM (1998) Water-energy dynamics, climate, and prediction of woody plant species richness: An interim general model. Journal of Biogeography, 25, 379-398.

O’Brien EM, Field R, Whittaker RJ (2000) Climatic gradients in woody plant (tree and shrub) diversity: water-energy dynamics, residual variation, and topography. Oikos, 89, 588-600.

Otto-Bliesner B, Brady E, Clauzet G, Thomas R, Levis S, Kothavala Z (2006) Last Glacial Maximum and Holocene Climate in CCSM3. Journal of Climate, 19, 2526-2544.

Price PW (1991) The plant vigor hypothesis and herbivore attack. Oikos, 62, 244-251.

Qu YH, Song G, Gao B, Quan Q, Ericson PGP, Lei FM (2015) The influence of geological events on the endemism of East Asian birds studied through comparative phylogeography. Journal of Biogeography, 42, 179-192.

Sandel B, Arge L, Dalsgaard B, Davies RG, Gaston KJ, Sutherland WJ, Svenning J-C (2011) The influence of Late Quaternary climate change velocity on species endemism. Science, 334, 660-664.

Schuldt A, Baruffol M, Böhnke M, Bruelheide H, Hädtle W, Lang AC, Nadrowski K, von Oheimb G, Voigt W, Zhou HZ, Assmann T (2010) Tree diversity promotes insect herbivory in subtropical forests of south-east China. Journal of Ecology, 98, 917-926.

Shen MW, Chen SB, Bi MJ, Chen WD, Zhou KX (2016) Relationships between geographic patterns of ant species richness and environmental factors in China. Acta Ecologica Sinica, 36, 7732-7739. (in Chinese with English abstract) [沈 梦伟, 陈圣宾, 毕孟杰, 陈文德, 周可新 (2016) 中国蚂 蚁丰富度地理分布格局及其与环境因子的关系. 生态学 报, 36, 7732-7739.]

Stein A, Gerstner K, Kreft H (2014) Environmental heterogeneity as a universal driver of species richness across taxa, biomes and spatial scales. Ecology Letters, 17, 866-880.

Svenning J-C, Eiserhardt WL, Normand S, Ordonez A, Sandel
B (2015) The influence of Paleoclimate on present day patterns in biodiversity and ecosystems. Annual Review of Ecology, Evolution, and Systematics, 46, 551-572.

Tews J, Brose U, Grimm V, Tielbörger K, Wichmann MC, Schwager M, Jeltsch F (2004) Animal species diversity driven by habitat heterogeneity/diversity: The importance of keystone structures. Journal of Biogeography, 31, 79-92.

Thomas CD, Bulman CR, Wilson RJ (2008) Where within a geographical range do species survive best? A matter of scale. Insect Conservation and Diversity, 1, 2-8.

Wenninger EJ, Inouye RS (2008) Insect community response to plant diversity and productivity in a sagebrush steppe ecosystem. Journal of Arid Environments, 72, 24-33.

Wisz MS, Pottier J, Kissling WD, Pellissier L, Lenoir J, Damgaard CF, Dormann CF, Forchhammer MC, Grytnes J-A, Guisan A, Heikkinen RK, Høye TT, Kühn I, Luoto M, Maiorano L, Nilsson M-C, Normand S, Öckinger E, Schmidt NM, Termansen M, Timmermann A, Wardle DA, Aastrup P, Svenning J-C (2013) The role of biotic interactions in shaping distributions and realised assemblages of species: Implications for species distribution modelling. Biological Reviews, 88, 15-30.

Xu RG (2007) Fauna Inner Mongolia. III. Inner Mongolia University Press, Hohhot. (in Chinese) [旭日干 (2007) 内 蒙古动物志. III. 内蒙古大学出版社, 呼和浩特.]

Xu RG (2015) Fauna Inner Mongolia. IV. Inner Mongolia University Press, Hohhot. (in Chinese) [旭日干 (2015) 内 蒙古动物志. IV. 内蒙古大学出版社, 呼和浩特.]

Xu RG (2016a) Fauna Inner Mongolia. V. Inner Mongolia University Press, Hohhot. (in Chinese) [旭日干 (2016a) 内 蒙古动物志. V. 内蒙古大学出版社, 呼和浩特.]

Xu RG (2016b) Fauna Inner Mongolia. VI. Inner Mongolia University Press, Hohhot. (in Chinese) [旭日干 (2016b) 内 蒙古动物志. VI. 内蒙古大学出版社, 呼和浩特.]

Zhang K, Lin SL, Ji YQ, Yang CX, Wang XY, Yang CY, Wang HS, Jiang HD, Harrison RD, Yu DW (2016) Plant diversity accurately predicts insect diversity in two tropical landscapes. Molecular Ecology, 25, 4407-4419.

Zhao YZ, Zhao LQ (2014) Key to the Vascular Plants of Inner Mongolia. Science Press, Beijing. (in Chinese) [赵一之, 赵 利清 (2014) 内蒙古维管植物检索表. 科学出版社, 北 京.]

Zhu H, Peng YY, Wang DL (2008) Effects of plant on insect diversity: A review. Chinese Journal of Ecology, 27, 2215-2221. (in Chinese with English abstract) [朱慧, 彭媛 媛, 王德利 (2008) 植物对昆虫多样性的影响. 生态学杂 志, 27, 2215-2221.]

Zobel M (1997) The relative role of species pools in determining plant species richness: An alternative explanation of species coexistence? Trends in Ecology and Evolution, 12, 266-269.

(责任编委: 陈圣宾 责任编辑: 间文杰) 